\title{
A pluripotent approach
}

A combination of protein and DNA isolation methods expands our grasp of cellular reprogramming.

Embryonic stem cell (ESC) pluripotency is maintained by a network of transcription factors (TFs) regulated by the master TFs OCT4, SOX2 and NANOG (collectively called OSN). Because TFs function on chromatin, biologically relevant information is more likely to be gained from the identification of factors associated with OSN in the DNA-bound state. With this in mind, Jeroen Krijgsveld of the German Cancer Research Center and his colleagues developed ChIP-SICAP (chromatin immunoprecipitation followed by selective isolation of chromatin-associated proteins).

Several approaches exist to isolate chromatin-associated complexes. In developing ChIP-SICAP, however, Krijgsveld and colleagues aimed to overcome two enduring hurdles: the discrimination between interactions occurring on and off chromatin, and the seemingly mundane yet critical issue of antibody contamination that can interfere with the detection of low-abundance proteins by mass spectrometry.

The initial step of the approach consists of classic ChIP using an antibody to the factor of interest. To place an affinity tag on the precipitated DNA, the researcher then treats the sample with terminal deoxynucleotidyl terminal transferase in the presence of biotinylated nucleotides. This is followed by denaturation under conditions that disrupt any non-cross-linked protein-protein and protein-DNA interaction before the DNA is isolated a second time on streptavidin-coated beads. Further stringent washes ensure that only complexes intimately associated with the target protein on chromatin remain bound; these complexes can then be identified by mass spectrometry. The DNA fragments released during the last step of the procedure are also suitable for high-throughput sequencing, which allows for complementary identification of the associated genomic binding sites.

Using ChIP-SICAP, the researchers identified over 400 proteins associated with chromatin-bound OSN in mouse ESCs. Among those not previously known to be involved in maintenance of the pluripotent state was TRIM24, an E3 ubiquitin ligase. TRIM24 was found to increase the efficiency of somatic cell reprogramming, demonstrating that ChIPSICAP could identify new OSN-associated pluripotency factors.

Although it has limitations such as the need for specific antibodies, ChIP-SICAP appears to be a promising tool for future explorations of transcriptional networks.

\section{Stéphane Larochelle}

\section{RESEARCH PAPERS}

Rafiee, M.-R. et al. Expanding the circuitry of pluripotency by selective isolation of chromatinassociated proteins. Mol. Cell 64, 624-635 (2016). 